\title{
Diagnóstico de componentes en la producción de textos expositivos en inglés
}

\section{Analysis of the components in written production of English expository texts}

\author{
Mónica Alejandra Logroño Becerra. ${ }^{1}$, Carmita Eulalia Rojas Castro. ${ }^{2}$ \& Ángel Paul Obregon \\ Mayorga. ${ }^{3}$ \\ Recibido: 10-09-2019 / Revisado: 03-10-209 /Aceptado: 04-11-2019/ Publicado: 05-12-2019
}

\begin{abstract}
DOI: https://doi.org/10.33262/cienciadigital.v3i4.2.1000

The main purpose of this research was to analyses the components in the production of expository texts in English by means of essays written by the students of sixth level group R of the Language Centre of the Higher Polytechnic School of Chimborazo during the academic period March-August 2017.This research was of field, descriptive, and bibliographic type. In first instance, ten of twelve teachers were evaluated in order to identify strategies to be considered in the intervention plan, which had a set of activities developed with the students during nine weeks. Then, the activities were evaluated by using a rubric under a Likert scale, validated by academic experts in the area, to be subsequently subjected to the Cronbach Alpha method. The sample was intentional, it was made of 32 students (men and women) belonging to the experimental group. The technique used was a survey by means of a questionnaire that evaluated the components of organization, coherence, cohesion and Grammar structure. The results obtained were tabulated, analyzed and interpreted through tables and graphs. Later a statistical test was applied, which determined that it was necessary to apply the intervention plan in the students and once applied, the production of expository texts was positively improved.
\end{abstract}

Keywords: Analysis, written production components, expository texts, English

\section{Resumen}

El objetivo de investigación fue diagnosticar los componentes en la producción de textos expositivos en inglés, a través de ensayos con los estudiantes de sexto nivel paralelo "B" de Inglés del Centro de Idiomas de la Escuela Superior Politécnica de Chimborazo en el período

1 Escuela Superior Politécnica de Chimborazo, Facultad de Administración de Empresas, Riobamba, Ecuador, ma_logrono@espoch.edu.ec

2 Escuela Superior Politécnica de Chimborazo, Facultad de Ciencias, Riobamba, Ecuador, crojasc@espoch.edu.ec

3 Escuela Superior Politécnica de Chimborazo, Facultad de Informática y Electrónica, Riobamba, Ecuador, paul.obregon@espoch.edu.ec 
marzo - agosto 2017. La investigación fue descriptiva, de campo y bibliográfica. En primera instancia se evaluaron a 10 de 12 docentes, con el fin de identificar las estrategias que debieron ser consideradas en el plan de intervención; el mismo que contenía un conjunto de actividades que se desarrollaron durante de nueve semanas. Las actividades fueron evaluadas mediante una rúbrica con escala de Likert, la misma que fue validada por expertos académicos y sometida al método de Alpha de Cronbach. La muestra fue intencional, compuesta por 34 estudiantes (hombres y mujeres) que representó al grupo de experimental. Se utilizó la técnica de la encuesta a través de un cuestionario, que evaluó los componentes de organización, Coherencia, Cohesión y estructuración. La evaluación fue aplicada al inicio y al final del módulo a la muestra de la investigación. Los resultados obtenidos fueron tabulados, analizados e interpretados mediante tablas y gráficos. Posterior se aplicó una prueba estadística, que determinó que fue necesario aplicar el plan de intervención en los estudiantes y una vez aplicado se mejoró positivamente la producción textos expositivos. Palabras claves: Análisis, written production components, expository texts, English

\section{Introducción}

La evaluación de textos y composiciones, se basaba casi totalmente en detectar los errores gramaticales, dejando de lado la conexión entre las frases, especialmente en textos complejos en los que la organización y coherencia de las ideas es fundamental. En la actualidad, este tipo no es totalmente dominante, aunque aún persiste, pues se ha empezado a poner cuidado en los conectores discursivos, pronombres, sustitución sinonímica, repetición léxica y otros recursos y herramientas desarrolladas por las investigaciones en lingüística del Texto.

En los 60, diferentes perspectivas teóricas y metodológicas se plantearon para reformular el modelo de la gramática generativa transformacional; que estudia el texto a dos niveles: superficial y profundo, de allí, los fundamentos teóricos de la lingüística del texto han ido experimentando paulatinamente un desplazamiento desde la comprensión del funcionamiento interno de las instancias textuales hasta su rendimiento en un mundo real de comunicación e interacción social, abarcando cada vez más aspectos como la participación de los interlocutores en la comunicación, la funcionalidad de los textos en diferentes contextos sociales y las bases psicológicas que sustentan los procesos de producción e interpretación de textos.

Posteriormente, se plantea que la comunicación trasciende los límites oracionales, es así que nace la lingüística del texto como un nuevo enfoque del lenguaje cuyos precursores fueron Teun van Dijk, Hannes Rieser, Janos Petófi, Jens Ihwe y Werner Kummer. El interés por explorar esta nueva tendencia se expandió desde Alemania Oriental y Occidental hacia otras regiones como Holanda, Hungría, Francia, Rusia, Italia, España e Inglaterra.

Para Montolío (2000) el texto expositivo es la base del discurso académico, ya que es la secuencia prototípica usada para comunicarse, analizar, y construir conocimiento. El texto expositivo o 
explicativo informa con el fin de hacer entender algo a alguien con una intención más didáctica, formal e incluso científica.

De acuerdo a Rollins (2009) el texto expositivo se basa en hechos y no en opiniones por lo que en muchos de los casos será necesario recurrir a varias fuentes de información que pueden ser primarias o secundarias. El texto expositivo es también conocido como informativo, cuyo propósito es explicar un tópico o tema.

El ensayo es una composición formal escrita con fines académicos, por esta razón debe ser escrito tomando en cuenta las reglas de organización y estructura, además de delimitar un tema, y considerar su audiencia. Al escribir ensayos en inglés, el estudiante debe usar todos los recursos de escritura y coordinar sus ideas ya que un ensayo efectivo no será posible sin la preparación y organización.

Para Conelly y Patrick (2012) el ensayo es una oportunidad para demostrar conocimiento, nivel intelectual y dominio del lenguaje. Al tratarse de una composición escrita formal, un ensayo debe mantener estructura sintáctica y semántica que los/las estudiantes de inglés deben ir desarrollando paulatinamente lo cual incluye aspectos como: lenguaje objetivo, gramática, oraciones completas, puntuación, por esta razón la estructura básica de un ensayo en inglés debe contener una introducción, desarrollo y conclusión.

Según Wingersky et al (2009), al desarrollar el párrafo introductorio, se deben tomar en cuenta ciertos aspectos como los mecanismos de atención (hook), su conexión por medio de transiciones y la tesis conocida también como idea principal o "thesis statement" en inglés.

El párrafo introductorio es el primer párrafo del ensayo, cuyo objetivo principal es capturar la atención del lector a la vez que le permite tener una visión de lo que todo el ensayo tratará y podrá decidir si la información subsiguiente es conveniente o no para sus intereses.

Moore y Lan (2011) reconocen que el sustento de la idea principal constituye la parte más larga del ensayo, porque encierra la evidencia que comprueba la validez de la idea principal del ensayo punto por punto; por lo que cada párrafo discutirá un aspecto particular empezando por una idea principal. La misión más importante de los párrafos que sustentan el ensayo es fundamentar la idea principal con información objetiva y detallada creando a su vez conexiones lógicas para dar un sentido general.

Para Oshima y Hogue (2006) la conclusión es el párrafo que se escribe al final del ensayo y replantea sus puntos más sobresalientes. La conclusión tiene tres propósitos fundamentales: Primero, señala la finalización del trabajo escrito, para lo cual utiliza frases clave (en conclusión, finalmente, etc.). Segundo, deja en claro lo más importante del ensayo a través de un resumen breve o de un parafraseo adecuado, y, tercero, si la conclusión es efectiva, permite que la audiencia reflexione sobre la idea principal del ensayo. 
Darwish (2010) considera a la cohesión como el "pegamento" que une los elementos del texto para conectarlos entre sí, y así dotarlo de unidad. Vista de este modo, la cohesión es la relación de interdependencia que presentan dos o más elementos de un texto pertenecientes a distintas oraciones.

Colby (2014) define a los elementos cohesivos como aquellos elementos que permiten la asociación lógica de ideas para aportar continuidad y unidad al texto. El autor señala la existencia de cinco elementos cohesivos que los describe como la clave para un texto cohesivo: Referencia, Elipsis, Sustitución (o sinonimia), transiciones y conjunciones.

Los recursos cohesivos facilitan la transmisión de ideas a través de la comunicación escrita. En el caso de la escritura en inglés como lengua extranjera; el correcto uso de los recursos cohesivos requiere de una práctica constante, sin embargo; existen casos en los que durante el proceso enseñanza-aprendizaje; éstos son analizados de manera superficial de modo que los y las estudiantes desconocen cómo usarlos, o simplemente memorizan algunos de ellos y gradualmente, el texto pierde su propósito comunicativo. Respecto a este punto, una cuestión a resaltar; es el hecho de que, para lograr un texto adecuadamente estructurado como producto final, es necesario contextualizar las actividades de escritura y de esta manera generar situaciones que demanden el uso significativo de recursos cohesivos.

La investigación fue viable, ya que se pudo contar con fuentes de información, existió la accesibilidad a los recursos materiales por parte de los investigadores. Además, se contó con el apoyo institucional para el uso de aulas e instalaciones y con la colaboración de los docentes, quienes contribuyeron con su tiempo y experticia en el área y finalmente los estudiantes quienes fueron partícipes activos en las diferentes actividades del proceso investigativo.

\section{Metodología}

La investigación tiene enfoque descriptivo, ya que en primera instancia analizó el criterio de los docentes para identificar los problemas y determinar estrategias. También analizó por medio de una rúbrica de evaluación la situación de los estudiantes en cuanto a los componentes para la producción de textos descriptivos en inglés. Por ello, se buscó los argumentos que sustenten la problemática encontrada.

La investigación fue de campo, porque los datos fueron obtenidos directamente de los estudiantes investigados; mientras que la observación del fenómeno de estudio se realizó en su mismo lugar de origen. La investigación tuvo carácter transversal ya que los datos fueron obtenidos en un tiempo límite de nueve semanas.

El estudio fue cuantitativo, pues se contó con un instrumento (cuestionario) donde los datos obtenidos fueron sometidos a la tabulación análisis e interpretación de información. Además, fue cualitativo porque fue necesario establecer preguntas y cuestiones que no son medibles en escala numérica 
La población estuvo conformada por 34 estudiantes de sexto nivel de inglés paralelo "B" del Centro de Idiomas de la ESPOCH, conformados por hombres y mujeres con edades entre 22 y 24 años que asistieron a clase en el período Marzo - agosto 2017, que provienen de las diferentes carreras que existentes en la institución.

La investigación es correlacional, en la que intervinieron componentes como variable independiente, que afectó a la producción de textos expositivos que representa la variable dependiente, Para su correlación se registraron datos cuantificados que fueron sometidos a una prueba estadista para determinar su efecto con la demostración de una hipótesis.

La experimentación tuvo una duración de 9 semanas en total (36 horas), donde se aplicó el plan de intervención que consistió en aplicar 4 horas para realizar las encuestas a los docentes; mientras que en las 36 horas clase, se desarrolló las actividades previstas, mismas que fueron evaluadas a través una rúbrica que considera las características o componentes atribuibles al texto expositivo como son: la organización, estructura, cohesión y coherencia.

Se optó por un modelo de progresión temática que derive en la enseñanza del idioma inglés, a fin de evaluar su efecto en la destreza de escritura para la producción de textos expositivos en inglés. Para ello, se inició con una encuesta dirigida a 10 docentes para conocer de sus estudiantes, sobre las dimensiones de la producción de los textos expositivos, a través de: delimitación del tema; problemática en coordinación de las ideas; conocimiento acerca del tema-rema; coherencia en la escritura; uso de ideas secundarias; progresión temática y uso de estrategias. El resultado de la encuesta permitió determinar la línea base o problemática que los estudiantes presentan en los componentes para la producción de textos expositivos.

Con el análisis de la encuesta dirigida a los docentes se diseñó y elaboró un plan de intervención que contemplo actividades para fortalecer los componentes de la producción de textos expositivos en inglés.

La secuencia de los textos expositivos consistió en una estructura que involucra los componentes principales como son: introducción, desarrollo y conclusión. Luego se procedió al estudio del texto a nivel de macro estructura y al análisis micro estructural del texto. Para ello, se adaptó patrones de textos expositivos de evaluaciones internacionales tipo ensayo tales como el Writing TOEFL IBT. Es decir, se efectuó el análisis de los componentes súper estructurales por separado, y proceder al análisis micro estructural en oraciones. Posterior se analizaron las oraciones por su importancia a fin de enlazar las ideas, con la ayuda de organizadores gráficos, que facilitaron la asimilación del objeto de estudio.

Para tener una mejor apreciación del nivel de logro alcanzado por los estudiantes, al final de cada actividad, se evaluó el logro de aprendizaje a través de actividades de retroalimentación y reflexión 
Se empleó la técnica de la encuesta. Para el análisis de información se recurrió a la lectura, al análisis documental, de contenido, sistematización e interpretación de datos, proveniente de los docentes y del grupo control:

Cada estudiante fue evaluado mediante una tarea a fin de obtener su calificación sobre 20 puntos,

La información obtenida fue tabulada a fin de registrar la afectación por cada una de las opciones de los 34 estudiantes de cada grupo.

Se sometió a una prueba estadística $\mathrm{Z}$ cuadrado que permitió comprobar o rechazar la hipótesis planteada en la investigación.

La validación, efectividad y confiabilidad del instrumento de medición fue supervisada por tres expertos del área, quienes realizaron sus aportes y observaciones y para garantizar la confiabilidad del instrumento se aplicó el método alpha de Crombach alcanzando un valor de 0.9624.

Las evaluaciones obtenidas de los estudiantes por medio del instrumento fueron realizadas en dos momentos (pre test y post test). El pre test se lo realizó antes de realizar el plan de intervención; mientras que el post test fue realizado luego de completado todas las actividades.

Los resultados fueron sometidos a la prueba estadística $\mathrm{Z}$ cuadrado mediante Microsoft Excel, el mismo que comparó las medias y desviación estándar de los dos test, permitiendo realizar las respectivas conclusiones de la investigación.

\section{Resultados}
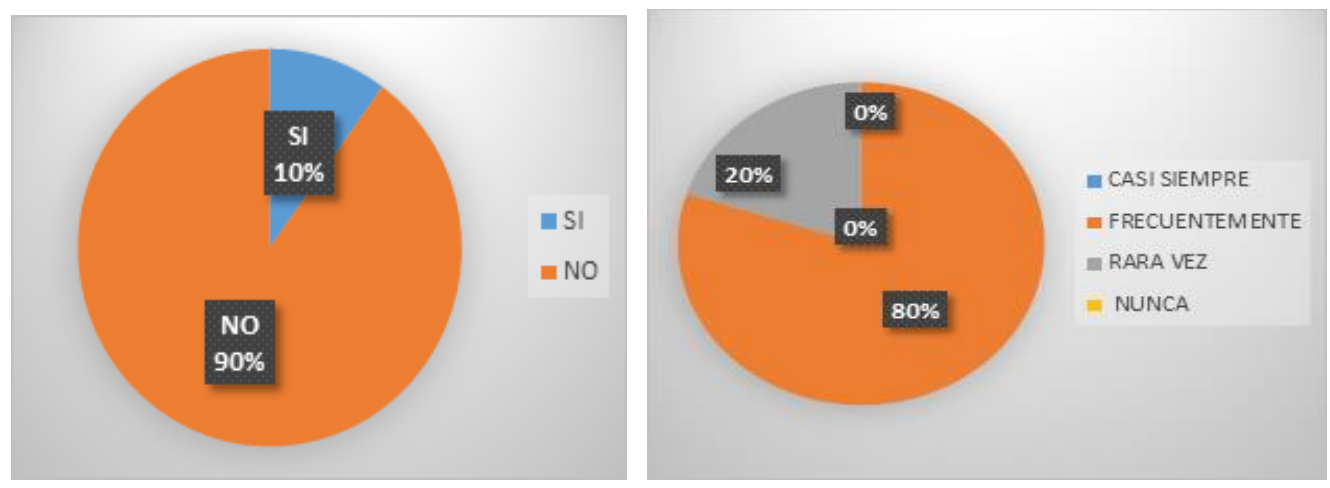

Figura 1. Resultados del Test

\section{Encuesta a Docentes}

En la siguiente tabla se visualizan los datos provenientes de las encuestas realizadas a 10, de los 12 profesores que imparten la asignatura de inglés en el sexto nivel del Centro de Idiomas de la ESPOCH. 
Pregunta 1. ¿Sus estudiantes delimitan un tema a través de la idea principal (thesis statement) en un texto cuando lo escriben?

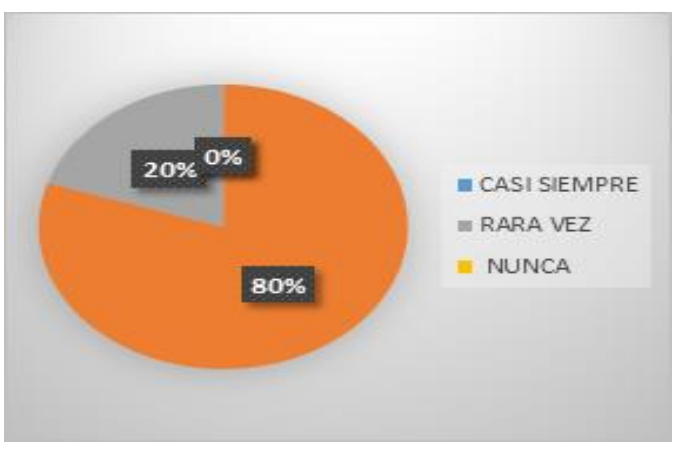

Figura 2. Delimitación de ideas principales

\section{Análisis e Interpretación.}

El 70\% de los docentes informó que los/las estudiantes delimitaban la idea principal al momento de escribir un ensayo básico; mientras que el 30\% reportó que los/las estudiantes no lo hacían. De acuerdo a estos resultados, la delimitación de la idea principal, sea ésta la topic sentence en el párrafo, o thesis statement en el ensayo; es un aspecto que aparentemente no representa mayor dificultad para los/las estudiantes de inglés, sin embargo; este es tan sólo el primer paso previo a la consecución del texto.

Pregunta 2. ¿Con qué frecuencia nota que sus estudiantes tienen problemas para coordinar sus ideas y transmitir información de manera lógica en sus textos escritos?

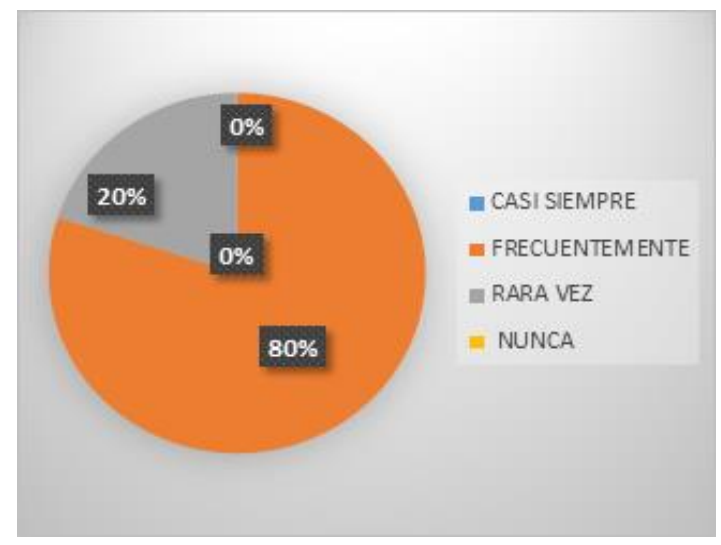

Figura 3. Problemas para coordinar ideas

\section{Análisis e Interpretación.}

En cuanto a problemas de coordinación de ideas y transmisión de información por parte de los/las estudiantes, el $80 \%$ de los/las docentes, informó que este hecho ocurría frecuentemente mientras 
que el 20\% reportó rara vez. Estos resultados demuestran que los problemas de coordinación impiden desarrollar una idea principal, por lo tanto, el texto carecerá de unidad y sentido, para evitar estos problemas, es necesario implementar actividades basadas en el uso de recursos cohesivos para lograr que la idea trascienda el nivel oracional y evolucione durante la producción de un texto hasta conseguir un todo coherente.

Pregunta 3. ¿Conoce sobre los componentes tema-rema en un texto en inglés?

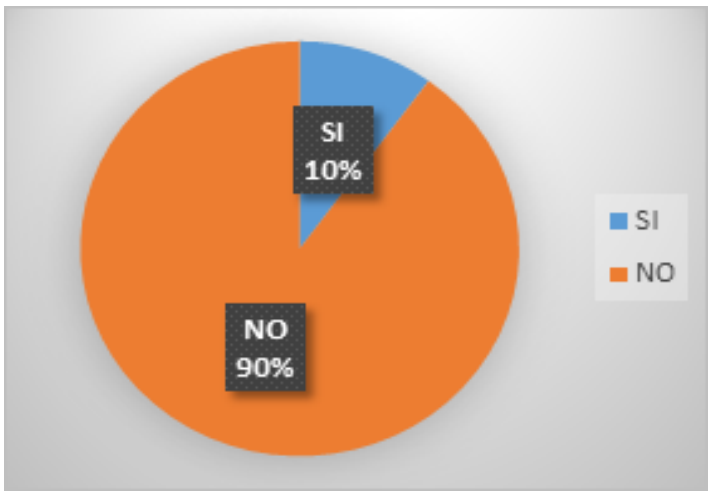

Figura 4. Componentes tema-rema

\section{Análisis e Interpretación.}

De un total de 10 docentes, uno de ellos está familiarizado con los componentes tema-rema. Este hecho revela que existen aspectos en el campo de la lingüística que no son lo suficientemente explorados, y que podrían contribuir en el proceso de enseñanza-aprendizaje de inglés como lengua extranjera.

Pregunta 4. ¿Conoce que es el proceso de tematización y su rol para lograr mayor coherencia en la escritura de un texto?

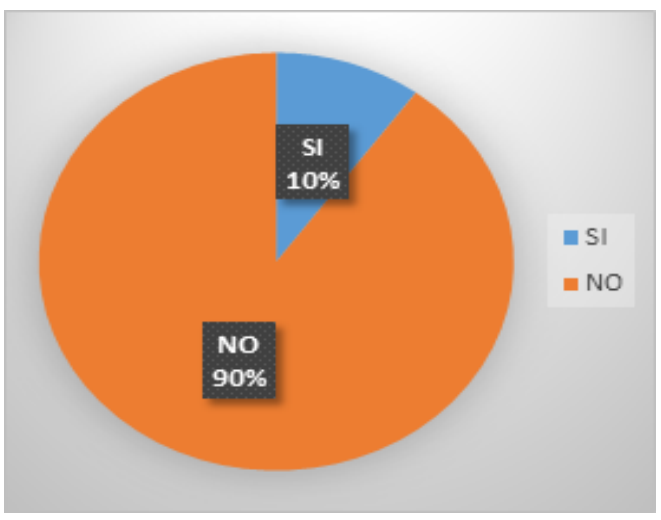

Figura 5. Coherencia en escritura 


\section{Análisis e Interpretación.}

Del total de 10 docentes, el $90 \%$ de ellos no conocen el proceso de tematización y su rol para lograr mayor coherencia en la escritura de un texto; mientras que el 10\% responde que Si. Los datos de la encuesta evidencian que la tematización es un proceso muy poco conocido por los/las docentes de la institución. Es decir, no aplican la progresión temática para organizar la información y coordinar ideas.

Pregunta 5. ¿Con qué frecuencia sus estudiantes usan ideas secundarias que sustenten la idea principal (Thesis Statement) de su ensayo básico?

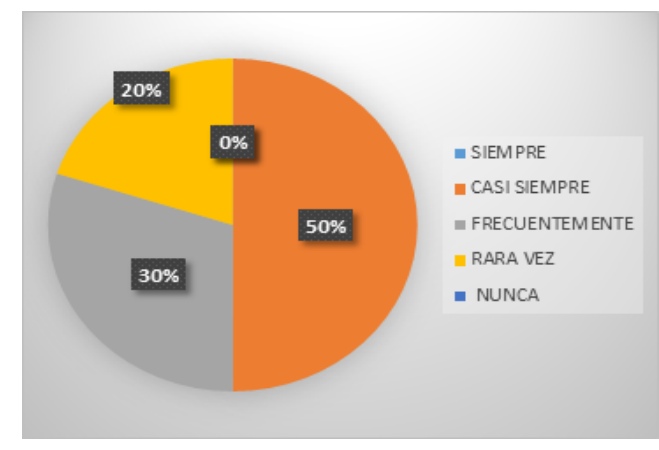

Figura 6. Ideas secundarias

\section{Análisis e Interpretación:}

Del total de los 10 docentes encuestados, $50 \%$ indica que los estudiantes Casi siempre, logran que usen ideas secundarias que sustenten la idea principal (Thesis Statement) de su ensayo básico; 30\% reportó Frecuentemente; el 20\% Rara vez; mientras que no se registraron frecuencias de las opciones Siempre y Nunca. Es decir que es posible desarrollar una idea principal, pero durante este proceso la coordinación de ideas se torna en una tarea compleja; lo que pone de manifiesto, que la existe el conocimiento de recursos cohesivos.; tan solo el $20 \%$ requiere atención de esta necesidad. Pregunta 6: ¿Ha escuchado acerca de la progresión temática y el modelo derivado como estrategia para mejorar la cohesión y coherencia en textos escritos en inglés?

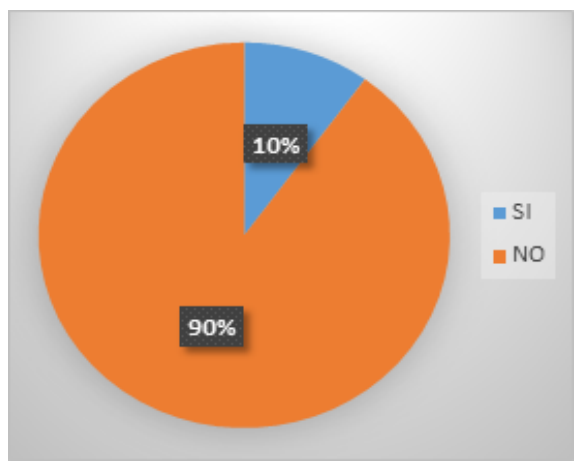

Figura 7. Progresión temática 


\section{Análisis e Interpretación:}

De los 10 docentes, el 90\% indica que No han escuchado acerca de la progresión temática y del modelo derivado como estrategia de mejora de la cohesión y coherencia; mie4ntras que el $10 \%$ indica que SI. Los resultados que se obtuvo en las preguntas 3, 4, y 6, reflejan que la Lingüística Textual es un área poco explorada. Se debe tomar en cuenta su efecto positivo en las propiedades de cohesión y coherencia en la producción escrita. En este caso la mayoría de estudiante requiere instruirse en la progresión temática.

Pregunta 7. ¿Considera que es necesario implementar estrategias que contribuyan a la producción escrita de sus estudiantes para lograr textos más cohesivos y coherentes en inglés?

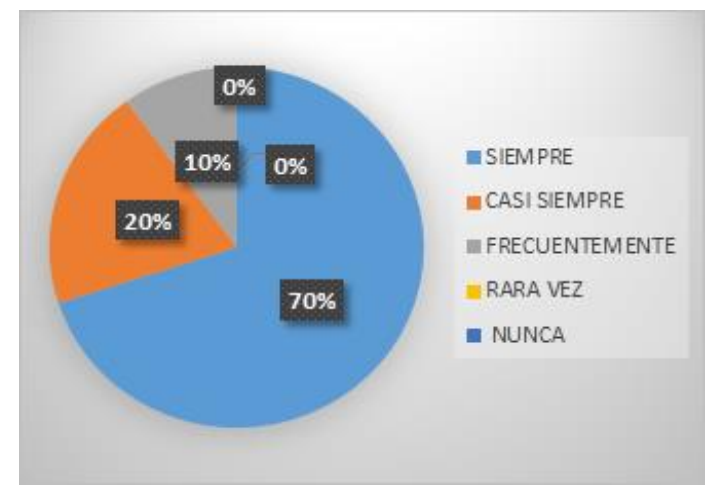

Figura 8. Implementación de estrategias

\section{Análisis e Interpretación:}

Del total de 10 docentes, el $70 \%$ indica que Siempre considera necesario implementar estrategias para la producción escrita de los estudiantes, para lograr cohesión y coherencia. Al ser la cohesión y la coherencia problemas frecuentemente identificados por los/las docentes de la institución, existe una gran necesidad de implementar recursos que ayuden a mejorar la unidad y continuidad del texto. Frente a esta necesidad la implementación de la progresión temática derivada como estrategia para desarrollar la competencia escrita en inglés contribuirá de manera significativa, lo cual justifica el presente estudio

Al ser la cohesión y la coherencia problemas frecuentemente identificados por los/las docentes de la institución, existe una gran necesidad de implementar recursos que ayuden a mejorar la unidad y continuidad del texto. Frente a esta necesidad la implementación de la progresión temática derivada como estrategia para desarrollar la competencia escrita en inglés contribuirá de manera significativa, lo cual justifica el presente estudio.

A continuación, se presentan los resultados de las evaluaciones obtenidas por los estudiantes del sexto nivel del Centro de Idiomas de la Escuela superior Politécnica de Chimborazo. Las calificaciones se obtuvieron mediante la aplicación de una de rúbrica para el diagnóstico de los 
componentes en la producción de textos expositivos en inglés (ensayo básico) creados por 34 estudiantes del paralelo "B". Los componentes evaluados fueron: organización, estructura, cohesión-progresión y coherencia. Cada elemento de la rúbrica contiene opciones que fueron ponderadas para la evaluación cuya sumatoria es equivalente a 20 puntos. Los puntajes obtenidos fueron tabulados y analizados mediante una hoja de cálculo.

Tabla 1. Consolidado de frecuencias por cada componente (Pre test - Post test)

\begin{tabular}{lllllllll}
\hline & Excelente Bueno & \multicolumn{3}{c}{ Aceptable Regular } \\
& PRE POST PRE POST PRE POST PRE POST \\
\hline Organización 1 & 19 & 9 & 9 & 9 & 4 & 11 & 2 \\
Estructura & 0 & 6 & 1 & 20 & 17 & 6 & 14 & 2 \\
Cohesión & 0 & 17 & 2 & 10 & 11 & 6 & 17 & 1 \\
Coherencia & 0 & 7 & 0 & 22 & 13 & 4 & 18 & 1
\end{tabular}

\section{Fuente: Test de Coherencia}

Elaborado por: Logroño Mónica, 2017

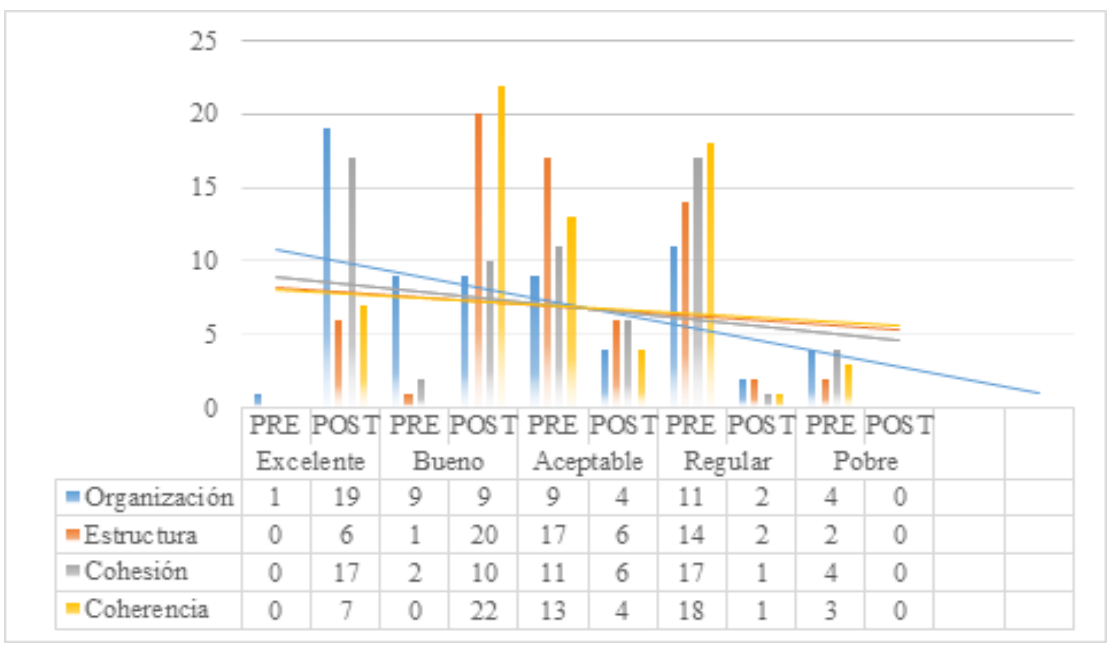

Figura 9. Consolidado de frecuencias por cada componente (Pre test-Post test)

Se puedo observar en el Gráfico 1, que las frecuencias se incrementaron notoriamente del post test en la opción Excelente en los diferentes componentes de la producción de los textos expositivos, realizados por los estudiantes del sexto nivel del Centro de Idiomas de la Escuela Superior Politécnica de Chimborazo. El post test en los diferentes componentes fueron fortalecidos positivamente en las opciones Excelente, Bueno y Aceptable.

Así mismo en el post test, la opción pobre tuvo frecuencias de cero; es decir que el plan de intervención fortaleció exitosamente los componentes de la producción textos expositivos. 
Además, se puedo observar que la línea de tendencia del componente de organización fue la que mejor fue desarrollada, seguida de la cohesión y cohesión.

\section{Análisis e Interpretación Estadística de Resultados}

A continuación, se describen la distribución de frecuencia, porcentajes, medias, desviación y varianza, coeficiente de correlación y la comprobación de la hipótesis.

\section{Cálculo de la desviación típica}

Tabla 2. Pre test

\begin{tabular}{|c|c|c|c|c|c|}
\hline$\underline{\mathbf{x}_{\mathbf{i}}}$ & $\mathbf{f}_{\mathbf{i}}$ & $\mathbf{x}_{\mathbf{i}} \mathbf{f}_{\mathbf{i}}$ & $\mathbf{x i}^{2}$ & $\mathbf{f}_{\mathrm{i}} \mathrm{x}_{\mathrm{i}}{ }^{2}$ & fr \\
\hline 0 & 0 & 0 & 0 & 0 & 0 \\
\hline 1 & 0 & 0 & 1 & 0 & 0 \\
\hline 2 & 0 & 0 & 4 & 0 & 0 \\
\hline 3 & 0 & 0 & 9 & 0 & 0 \\
\hline 4 & 1 & 4 & 16 & 16 & 2,9 \\
\hline 5 & 4 & 20 & 25 & 100 & 11,8 \\
\hline 6 & 4 & 24 & 36 & 144 & 11,8 \\
\hline 7 & 2 & 14 & 49 & 98 & 5,9 \\
\hline 8 & 4 & 32 & 64 & 256 & 11,8 \\
\hline 9 & 2 & 18 & 81 & 162 & 5,9 \\
\hline 10 & 5 & 50 & 100 & 500 & 14,7 \\
\hline 11 & 4 & 44 & 121 & 484 & 11,8 \\
\hline 12 & 3 & 36 & 144 & 432 & 8,8 \\
\hline 13 & 1 & 13 & 169 & 169 & 2,9 \\
\hline 14 & 0 & 0 & 196 & 0 & 0 \\
\hline 15 & 2 & 30 & 225 & 450 & 5,9 \\
\hline 16 & 0 & 0 & 256 & 0 & 0 \\
\hline 17 & 1 & 17 & 289 & 289 & 2,9 \\
\hline 18 & 1 & 18 & 324 & 324 & 2,9 \\
\hline 19 & 0 & 0 & 361 & 0 & 0 \\
\hline 20 & 0 & 0 & 400 & 0 & 0 \\
\hline & 34 & 320 & 2870 & 3424 & \\
\hline & & \multicolumn{4}{|c|}{$\sum \mathbf{f}_{\mathrm{i}} \mathbf{x}_{\mathrm{i}}^{2}$} \\
\hline
\end{tabular}

Fuente: Nivel de Confianza

Elaborado por: Autor 
Tabla 3. Nivel de Confianza-Pre test

\begin{tabular}{lr}
\hline Datos & \\
\hline Media: & 9,41 \\
Desv est: & 3,48 \\
Confianza: & $95 \%$ \\
N. Casos: & 34 \\
alfa: & $5 \%$ \\
Intervalo & $1,17 \quad 8,24$ a 10.58 \\
\hline
\end{tabular}

Fuente: Nivel de Confianza

Elaborado por: Autor

Tabla 4. Post test

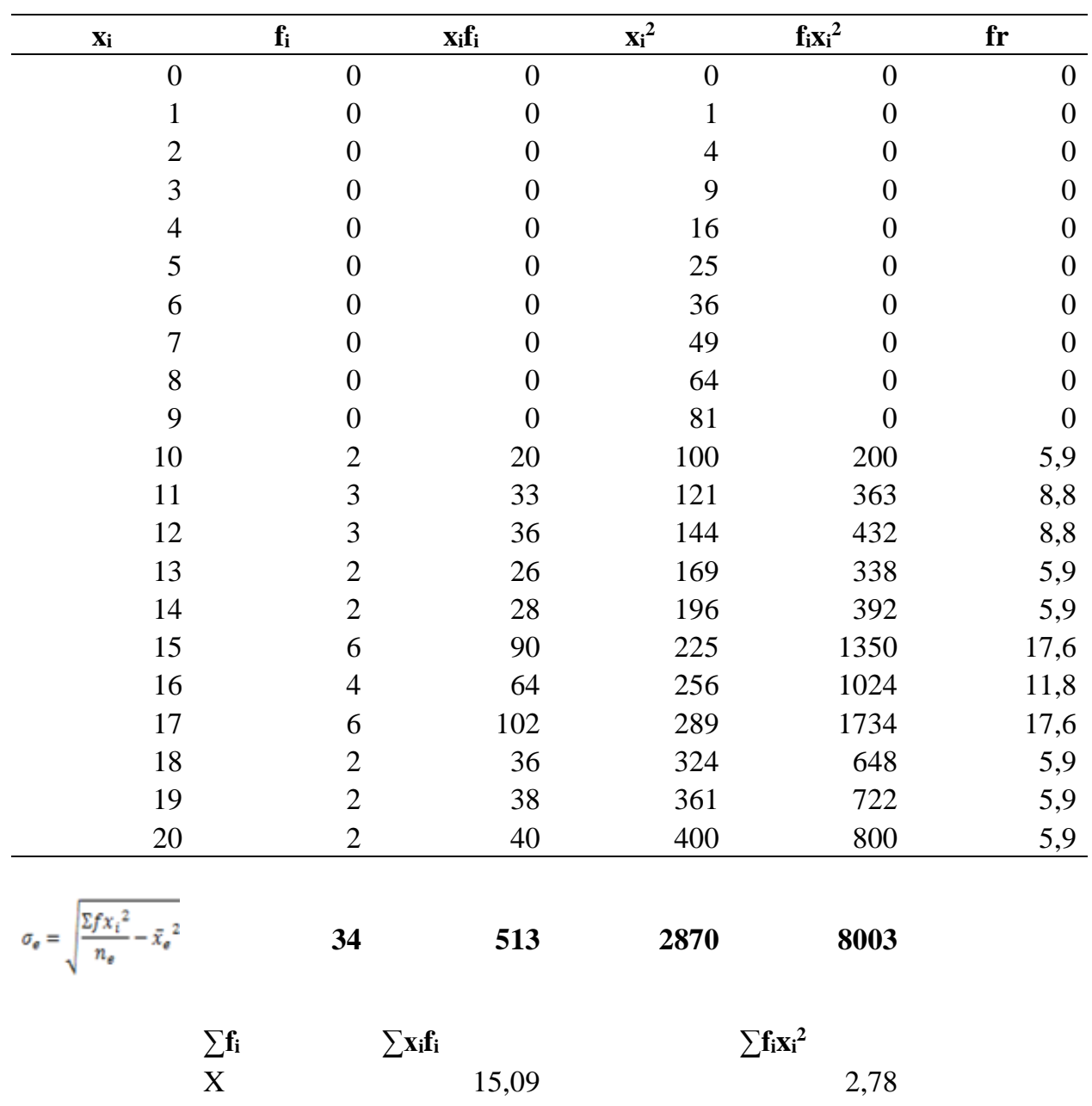

Fuente: Nivel de Confianza

Elaborado por: Autor 
Tabla 5. Nivel de Confianza-Pre test

\begin{tabular}{lr}
\hline Datos & \\
\hline Media: & 15,09 \\
Desv est: & 2,78 \\
Confianza: & $95 \%$ \\
N. Casos: & 34 \\
alfa: & $5 \%$ \\
Intervalo & $1,17 \quad 8,24$ a 10.58 \\
\hline
\end{tabular}

Fuente: Nivel de Confianza

Elaborado por: Autor

Coeficiente de Correlación

Donde:

$$
r=\frac{N \sum x y-\sum x \sum y}{\sqrt{\left[N \sum x^{2}-\left(\sum x\right)^{2}\right]\left[N \sum y^{2}-\left(\sum y\right)^{2}\right]}}
$$

$\mathrm{r}=$ coeficiente de correlación de Pearson.

Sxy = sumatoria de los productos de ambas variables.

$\mathrm{Sx}=$ sumatoria de los valores de la variable

independiente.

Sy $=$ sumatoria de los valores de la variable dependiente.

Sx2 = sumatoria de los valores al cuadrado de la variable independiente.

Sy2 = sumatoria de los valores al cuadrado de la variable dependiente.

$\mathrm{N}$ = tamaño de la muestra en función de parejas. 
Tabla 6. Coeficiente de correlación

\begin{tabular}{|c|c|c|c|c|c|}
\hline N. caso & VI (X) & VD (Y) & $\mathrm{X}^{2}$ & $\mathbf{Y}^{2}$ & $\mathbf{X Y}$ \\
\hline 1 & 4 & 14 & 16 & 196 & 56 \\
\hline 2 & 11 & 17 & 121 & 289 & 187 \\
\hline 3 & 12 & 15 & 144 & 225 & 180 \\
\hline 4 & 13 & 19 & 169 & 361 & 247 \\
\hline 5 & 10 & 17 & 100 & 289 & 170 \\
\hline 6 & 6 & 12 & 36 & 144 & 72 \\
\hline 7 & 5 & 11 & 25 & 121 & 55 \\
\hline 8 & 5 & 14 & 25 & 196 & 70 \\
\hline 9 & 8 & 17 & 64 & 289 & 136 \\
\hline 10 & 7 & 15 & 49 & 225 & 105 \\
\hline 11 & 10 & 19 & 100 & 361 & 190 \\
\hline 12 & 6 & 10 & 36 & 100 & 60 \\
\hline 13 & 7 & 11 & 49 & 121 & 77 \\
\hline 14 & 10 & 17 & 100 & 289 & 170 \\
\hline 15 & 12 & 16 & 144 & 256 & 192 \\
\hline 16 & 6 & 11 & 36 & 121 & 66 \\
\hline 17 & 5 & 15 & 25 & 225 & 75 \\
\hline 18 & 8 & 13 & 64 & 169 & 104 \\
\hline 19 & 11 & 13 & 121 & 169 & 143 \\
\hline 20 & 6 & 16 & 36 & 256 & 96 \\
\hline 21 & 10 & 18 & 100 & 324 & 180 \\
\hline 22 & 9 & 15 & 81 & 225 & 135 \\
\hline 23 & 9 & 17 & 81 & 289 & 153 \\
\hline 24 & 8 & 20 & 64 & 400 & 160 \\
\hline 25 & 11 & 12 & 121 & 144 & 132 \\
\hline 26 & 15 & 16 & 225 & 256 & 240 \\
\hline 27 & 12 & 12 & 144 & 144 & 144 \\
\hline 28 & 15 & 16 & 225 & 256 & 240 \\
\hline 29 & 8 & 15 & 64 & 225 & 120 \\
\hline 30 & 10 & 17 & 100 & 289 & 170 \\
\hline 31 & 5 & 10 & 25 & 100 & 50 \\
\hline 32 & 11 & 15 & 121 & 225 & 165 \\
\hline 33 & 17 & 18 & 289 & 324 & 306 \\
\hline 34 & 18 & 20 & 324 & 400 & 360 \\
\hline & 274 & 460 & 2690 & 7054 & 4175 \\
\hline
\end{tabular}

Coeficiente Correlación = 0, 54 (Correlación positiva moderada)

\section{Prueba de Hipótesis}

H1: El fortalecimiento de los componentes tiene un efecto significativo sobre la producción de textos expositivos en el Idioma Inglés.

Ho: El fortalecimiento de los componentes no tiene un efecto significativo sobre la producción de textos expositivos en el Idioma Inglés. 


\section{Lenguaje matemático}

$$
\begin{gathered}
\mathrm{Hi}: \bar{x}_{\text {post }} \neq \bar{x}_{\text {pre }}: \text { con A1: } \bar{x}_{\text {post }}>\bar{x}_{\text {prec }} \circ \mathrm{A} 2: \bar{x}_{\text {post }}<\bar{x}_{\text {pre }} \\
\text { Ho: } \bar{x}_{\text {post }}=\bar{x}_{\text {pre }}
\end{gathered}
$$

\section{GRUPOn PromedioDesv Est.}

Pre Test $349,41 \quad 3,48$

Post Test 3415,09 2,78

\section{Determinación de Valores Críticos y Zonas de Rechazo.}

Mediante el cálculo de la prueba paramétrica $\mathrm{Z}$ se rechaza la hipótesis nula si:

O también

Donde, es el valor teórico de Z para un nivel de significación del 5\%, a=0,05; es decir que la investigación tendrá un $95 \%$ de confiabilidad; caso contrario se acepta la hipótesis de investigación con una de las dos alternativas.

$$
\begin{aligned}
& \overline{x_{\text {post }}}=15.09 \quad \overline{x_{\text {pre }}}=9.41 \quad \sigma_{\text {post }}=2.78 \quad \sigma_{\text {pre }}=3.48 \quad n_{\text {post }}=34 \quad n_{\text {pre }}=34 \\
& Z=\frac{\overline{x_{\text {post }}}-\overline{x_{\text {pre }}}}{\sqrt{\frac{\sigma_{\text {post }}{ }^{2}}{n_{\text {post }}}+\frac{\sigma_{\text {pre }}{ }^{2}}{n_{\text {pre }}}}} \\
& Z_{c}=7.43 \\
& Z_{C}>Z_{T} \\
& 7.43>1,96
\end{aligned}
$$

Con un nivel de significación de 0,05 equivalente al $+/-1,96$, se rechaza la hipótesis nula Ho, ya que la solución Zc=7.43 se encuentra en el intervalo de la región de rechazo de Ho. En consecuencia, se acepta la hipótesis alternativa $\mathrm{H}_{1}$.

\section{Conclusiones}

El diagnóstico realizado a los docentes que participaron en la presente investigación, permitió identificar y ratificar los parámetros que definen la rúbrica de evaluación. Estos parámetros representan los componentes en la producción de textos expositivos en inglés que han sido sustentados mediante una revisión de literatura. De igual manera contribuyó en el diseño y 
elaboración de las actividades contenidas dentro del plan de intervención. El mismo que fue fundamental para fortalecer los componentes de organización, estructura, coherencia y cohesión para la producción de textos expositivos.

El análisis de los resultados de los componentes en la producción de textos expositivos en inglés, evidenció la existencia de problemas de deficiencias en su aplicación entre ellas: una escritura con lenguaje objetivo, gramatical, oraciones completas y puntuación; la composición escrita formal, manteniendo una estructura sintáctica y semántica compuesta de introducción, desarrollo y conclusión; la relación de interdependencia que presentan dos o más elementos de un texto pertenecientes a distintas oraciones; y la cualidad y propósito del texto escrito

Al relacionar los datos más representativos de los indicadores cohesión y estructura se puede evidenciar que los/las estudiantes experimentan problemas en el curso comunicativo del texto escrito. Es decir, la estructura gramatical es aceptable, pero la asociación de estas estructuras individuales entre ese sí no ocurre de manera efectiva por lo que el texto, se mantiene a nivel oracional. Esta relación a su vez, demuestra la importancia del proceso de tematización en la evolución del texto.

En un inicio al evaluar los componentes de la producción de textos expositivos, a través de la aplicación de la rúbrica, se obtuvo resultados que permitieron determinar las opciones que predominaron fueron: Pobre, Regular y Aceptable con un promedio del 96\%; la opción Bueno entre el 3 y 6\%; mientras que la opción Excelente no registro frecuencias; por lo que fue un escenario o línea base que propicio para el desarrollo de investigaciones, la misma que requirió el planteamiento de una propuesta con una estrategia mediante un plan de intervención, que sirvió de guía para el docentes y estudiantes, a fin de mejorar la producción de textos expositivos en inglés.

Luego del plan de intervención, mejoraron sustancialmente las frecuencias en los componentes, siendo favorables las opciones de Excelente, Bueno y Regular, siendo claramente visualizadas en las líneas de tendencia favorables, Por otro lado, a opción pobre presentó cero frecuencias; datos que estadísticamente demostraron que el fortalecimiento de los componentes contribuyó positivamente en la producción de textos expositivos como se demostró mediante el análisis estadístico.

\section{Referencias bibliográficas}

Darwish, Ali. (2003). Elements of Translation. Melbourne. second edition

Colby, H. (2014). Wham Media's 6 secrets to Ielts Academic Writing Sucess. London: Wham Media.

Conelly, J., \& Patrick, F. (2012). Essay writing skills. London: Kogan Page. 
Thwe, Jens, Linguistik in der Literaturwissenschaft, Munich, Bayerischer Schulbuch Verlag (1972).comp., Literaturwissenschaft und Linguistik, 3 tomos, Frankfurt, Athenaum, $1971 / 1972$.

Hannes, \& Rieser, (1973) Studies in text grammar, Dordrecht, Reidel.

Kummer, Werner, Grundlagen der Texttheorie, Reinbek/Hamburgo, Rowohlt, 1975

Montolío. (2000). Manual práctico de la Escritura Académica. Barcelona: Ariel.

Moore, k., \& Lan Cassel, S. (2011). Techniques for College Writing: The thesis Statement and Beyond. USA: Wadsworth. Cengage Learning.

Oshima, A., \& Hogue, A. (2006). Writing Academic English. N.Y: Pearson Longman.

Rieser, Hannes (1978) "El desarrollo de la gramática textual" en Janes Petófí y Antonio García Berrio(eds) Lingüística del texto y crítica literaria, Madrid, Alberto Corazón.

Rollins, B. V. (2009). The Great Book of Writing. San Diego; CA: classroomcomplete press.

VAN DIJK, TEUN A., Estructuras y funciones del discurso. Una introducción interdisciplinaria a la lingüística del texto y a los estudios del discurso, Siglo XXI Editores, México, 1980.

Teun A. Van Dijk, János Petófí y M. A. K. Halliday", Fuentes Humanísticas 43, Lingüística, págs. $131-144$.

Wingersky, J., Janice, K., Boerner, D., \& Balog, H. (2009). writing Paragraphs and Essays: Integrating Reading, and Grammar skills. USA: Wadsworth.

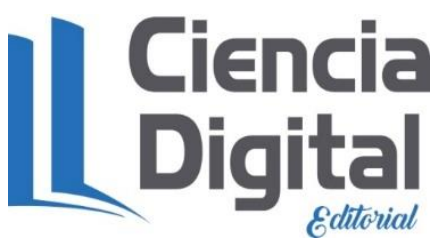




\section{PARA CITAR EL ARTÍCULO INDEXADO}

Logroño Becerra, M., Rojas Castro, C., \& Obregon Mayorga, Ángel. (2019). Diagnóstico de componentes en la producción de textos expositivos en inglés. Ciencia Digital, 3(4.2), 06 - 24. https://doi.org/10.33262/cienciadigital.v3i4.2.1000

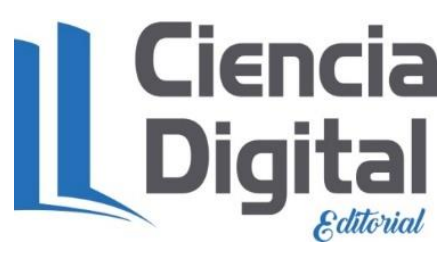

El artículo que se publica es de exclusiva responsabilidad de los autores y no necesariamente reflejan el pensamiento de la Revista Ciencia Digital.

El artículo queda en propiedad de la revista y, por tanto, su publicación parcial y/o total en otro medio tiene que ser autorizado por el director de la Revista Ciencia Digital.
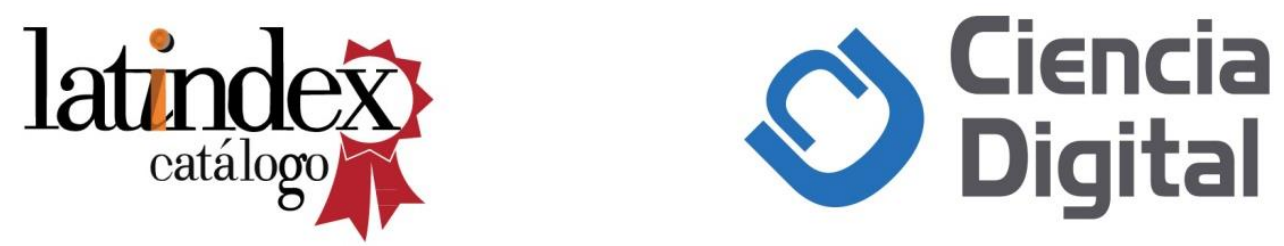